\title{
Synthesis of New Organophosphorus Compounds Derived from 2-Arylidenecyclohexan-1-ones
}

\author{
Mona Arsanious * and Ibtisam Hennawy \\ Department of Organometallic and Organometalloid Chemistry \\ National Research Centre, 33 El- Bahouth Street Dokki, P.O. Box 12622, Giza, Egypt
}

\begin{abstract}
Arylidene)cyclohexane-1-one derivatives react with 2,4- bis (phenylthio) -1,3-dithia,2,4diphosphetane-2,4-disulphide (JR) to give a mixture of the benzodithiadiphosphi-nine -2-sulphides and dithiadispirotetradecanes. The same reaction was carried out using Lawesson's reagent to yield the 4-methoxyphenylbenzodithiaphosphinine sulfides. A putative reaction mechanism involves an initial thiation of arylidenecyclohexane-1-one derivatives then Michael addition by sulfide anion of Lawesson's reagents followed by intramolecular cyclization, and resulting in the formation of new heterocyclic compounds containing phosphorus moiety. Biological activities for some of the new compounds are also reported.
\end{abstract}

Keywords: Japanese and Lawesson's reagents; Phosphorus Compounds; Cyclohexan-1-one derivatives.

\section{Introduction}

Arylidene cyclohexanones attract great attention of many investigators effective class of $\alpha-\beta$ unsaturated carbonyl compound ${ }^{1}$; for synthesis of saturated and partially saturated heterocyclic ring systems. $^{2-4}$ Diverse biological and pharmacological properties were also reported for these analogues as antimicrobial $^{4,5}$, antitubercular ${ }^{6}$, antioxidant ${ }^{7}$, antiangiogenic $^{8,9}$, cytotoxic ${ }^{10,11}$, cholesterol-lowering ${ }^{12}$, pesticidal ${ }^{13}$, and HIV- integrate inhibitory ${ }^{14}$ activities. Additionally, phosphorus compounds such as Japanese (JR, 2a) and Lawesson's reagents $(\mathrm{LR}, \mathbf{2 b})$ are considered as selective reagents in the realm of sulfur chemistry due to their ability to convert the carbonyl groups into their thiocarbonyl functions ${ }^{15}$. They can also be utilized in the construction of heterocycles containing either sulfur or phosphorus atoms which are of particular interest in industrial and pharmacological fields ${ }^{16-19}$.

This together with our interest in the organophosphorus chemistry of carbocyclic and heterocyclic compounds $4,26,27$ have prompted the present study directing towards investiga-tion of the behavior of 2-arylidenecyclohexanones 1a,b with 2a,b (Scheme 1). Antimicrobial properties of the newly synthesized compounds will be also considered against Gram- positive, Gram-negative and fungi.<smiles>[R]c1cc([R])c(C=C2CCCCC2=O)cc1[R]</smiles>

$$
\begin{aligned}
& \text { 1a; } R=\mathbf{N}\left(\mathrm{CH}_{3}\right)_{2}, \mathbf{R}^{1}=\mathbf{H} \\
& \text { 1b; } R=\mathbf{C l}, \mathbf{R}^{\mathbf{1}}=\mathbf{H} \\
& \text { 1c; } R=\mathbf{H}, \mathbf{R}^{\mathbf{1}}=\mathrm{OCH}_{3}
\end{aligned}
$$

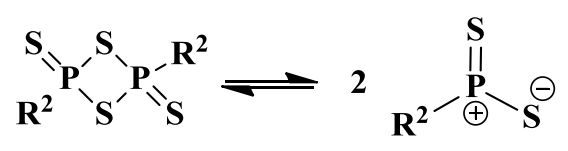

$2 \mathrm{a} ; \mathbf{R}^{2}=\mathrm{SC}_{6} \mathrm{H}_{5}$ $2 b ; \mathbf{R}^{2}=4-\mathrm{C}_{6} \mathrm{H}_{4} \mathrm{OCH}_{3}$

Scheme 1. Starting materials 


\section{Experimental Section}

\section{Materials and equipment}

Melting points were determined in open glass capillaries using an Electrothermal IA 9100 series digital melting point apparatus (Electrothermal, Essex, U.K.). IR spectra were recorded ( $\mathrm{KBr}$ pellets) on a Perkin- Elmer 1650 FT-IR spectrophotometer. The ${ }^{1} \mathrm{H}$-, and ${ }^{13} \mathrm{C}$-NMR spectra were recorded in $\mathrm{CDCl}_{3}$ on a Joel spectrometer $\left({ }^{1} \mathrm{H}: 500,{ }^{13} \mathrm{C}: 125\right.$ $\mathrm{MHz})$. Chemical shifts were recorded in $\delta$ values relative to TMS as internal reference. The coupling constants $(J)$ are given in Hertz $(\mathrm{Hz})$. Mass spectra were recorded at $70 \mathrm{eV}$ on a JEOL JMS/AX-500 spectrometer.

Cyclohexanone, 4-chlorobenzaldehyde, and 4dimethylaminobenzaldehyde were obtained from Sigma-Aldrich. Thiophenol, and anisole from Merck AR grade.

General procedure for the synthesis of 3a-c, 4a-c and 5a-b

A mixture of equimolar amounts of 2 arylidenecyclohexanone 1a,b (1mmol) and 1,3,2,4dithiadiphosphetane-2,4-disulfide $\mathbf{2 a}$ /or $\mathbf{2 b}$ was refluxed for $1 \mathrm{~h}$ in $30 \mathrm{ml}$ dry toluene. After removal of toluene under reduced pressure, the given crude material was subjected to column chromatography, using ethyl acetate / petroleum ether $\left(40-60{ }^{\circ} \mathrm{C}\right)$ as an eluent giving 3a-c, 4a-c, 5a,b.

\section{4-[4-(Dimethylamino)phenyl]-5,6,7,8-tetrahydro-} 4H-1,3,2-benzodithiaphosphinin-2-sulfide 3a

Eluent solution : ethyl acetate / petroleum ether (40$\left.60{ }^{\circ} \mathrm{C}\right) ; 45: 55(\mathrm{v} / \mathrm{v})$.

Yield: $60 \% ; \mathrm{mp}=171^{\circ} \mathrm{C}$

FT- IR (KBr) v, cm-1: 2910, $2854\left(\mathrm{CH}_{2}\right.$ stretching), 1170 (P-S- stretching), $1650(\mathrm{C}=\mathrm{C}$, stretching), 680 ( $\mathrm{P}=\mathrm{S}$, stretching).

$\mathrm{H}^{1} \quad \mathrm{NMR}\left(\mathrm{CDCl}_{3}, 500 \mathrm{MHz}\right)$ ( $\left.\delta: \mathrm{ppm}\right):$ 1.79$1.76\left(\mathrm{~m}, 2 \mathrm{H}, J=2.3, \mathrm{CH}_{2}\right), 1.90-1.88(\mathrm{~m}, 2 \mathrm{H}, J=2.3$ , $\left.\mathrm{CH}_{2}\right), 2.51-2.86\left(\mathrm{t}, 4 \mathrm{H}, J=3.5,2 \mathrm{CH}_{2}\right), 3.00[\mathrm{~s}, 6 \mathrm{H}$, $\left.\mathrm{N}\left(\mathrm{CH}_{3}\right)_{2}\right], 4.39\left(\mathrm{~d}, 1 \mathrm{H},{ }^{3} J_{H P}=4.4, \mathrm{HC}-\mathrm{S}-\mathrm{P}\right), 6.70-$ 6.67 (d, 2H, $J=8.4$, aromatic), 7.40-7.38 (d, 2H, $J=$ 8.4 , aromatic), $7.53(\mathrm{~d}, 2 \mathrm{H}, J=8.4$, aromatic), 7.737.75 (m, 3H, aromatic)

${ }^{13} \mathrm{C}$ NMR: $\left(\mathrm{CDCl}_{3}, 125 \mathrm{MHz}\right) \quad(\delta: \mathrm{ppm}) 34.1(d$, ${ }^{2} J_{C P}=29.02$, HC-S-P), 22.9, 24.4, 24.8, $23.9\left(\mathrm{CH}_{2}\right)$, 41.7 $\left(\mathrm{N}-\mathrm{CH}_{3}\right), \quad 112.7,125.8,127.1, \quad 127.8$, 128.9,131.0,132.6, 148.0 (C-aromatic).

Anal.Calc. for $\mathrm{C}_{21} \mathrm{H}_{24} \mathrm{NPS}_{4} \mathrm{C}, 56.09 \%$, H 5.38\%, N, $3.11 \%$, P, 6.89\%, S, $28.52 \%$. Found: C, 56.30\%, H, $5.54 \%, \mathrm{~N}, 3.25 \%, \mathrm{P}, 6.90 \%, \mathrm{~S}, 28.32 \%$.

\section{4-[4-Chlorophenyl]-5,6,7,8-tetrahydro-4H-1,3,2- benzodithiaphosphinin-2-sulfide $3 \mathrm{~b}$}

Eluent solution : ethyl acetate / petroleum ether (40$\left.60{ }^{\circ} \mathrm{C}\right) ; 60: 40(\mathrm{v} / \mathrm{v})$.

Yield $62 \%, \mathrm{mp}=183^{\circ} \mathrm{C}$.

FT-IR (KBr) v, cm-1: 2990, $2845\left(\mathrm{CH}_{2}\right.$ stretching), 750 $(\mathrm{C}-\mathrm{Cl}), 665(\mathrm{P}=\mathrm{S}), 1540,1665(\mathrm{C}=\mathrm{C}-$, stretching of aromatic), 1150 (P-S-).
$\mathrm{H}^{1} \mathrm{NMR}\left(\mathrm{CDCl}_{3}, 500 \mathrm{MHz}\right) \quad$ ( $\left.\delta: \mathrm{ppm}\right):$ 1.71-1.63 (m, 2H, $\left.\mathrm{CH}_{2}\right), 1.95-1.81\left(\mathrm{~m}, 2 \mathrm{H}, \mathrm{CH}_{2}\right), 2.01,2.48$ (t, $\left.4 \mathrm{H}, J=3.6,2 \mathrm{CH}_{2}\right), 4.21\left(\mathrm{~d}, 1 \mathrm{H},{ }^{3} J_{H P}=3.9, \mathrm{CH}-\mathrm{S}-\mathrm{P}\right)$, 6.70-6.67 (d, 2H, $J=8.4$, aromatic), 7.40-7.38 (d, $2 \mathrm{H}, J=8.4$, aromatic),7.73-7.74 (d, 2H, $J=8.0$, aromatic),7.75-7.76 (m, 3H, aromatic).

${ }^{13} \mathrm{C} \mathrm{NMR}\left(\mathrm{CDCl}_{3}, 125 \mathrm{MHz}\right) \quad(\delta: \mathrm{ppm}): 34.7$ $\left(d, J_{C P}=26.02\right.$, HC-S-P $), 22.9,24.4,24.8,27.4$ $\left(\mathrm{CH}_{2}\right), 112.7,125.8,127.3,127.6,128.7,131.4$, 132.4, 148.3(C-aromatic).

Anal.Calc for $\mathrm{C}_{19} \mathrm{H}_{18} \mathrm{ClPS}_{4} \mathrm{C}, 51.74 \%, \mathrm{H} 4.11 \%, \mathrm{Cl}$, $8.04 \%$, P, 7.02\%, S, $29.08 \%$. Found: C,51.97\%, H, $4.41 \%, \mathrm{Cl}, 8.33 \%, \mathrm{P}, 7.42 \%, \mathrm{~S}, 13.32 \%$.

\section{4-[2,5-Dimethoxyphenyl]-5,6,7,8-tetrahydro-4H-}

\section{1,3,2-benzodithiaphosphinin-2-sulfide 3c}

Eluent solution : ethyl acetate / petroleum ether (40$\left.60{ }^{\circ} \mathrm{C}\right) ; 55: 45(\mathrm{v} / \mathrm{v})$.

Yield $70 \%, \mathrm{mp}=178{ }^{\circ} \mathrm{C}$.

FT- IR (KBr) v, cm-1: 2960,2840 ( $\mathrm{CH}_{2}$ stretching ), 1180 (P-S-), 1650(C=C, aromatic), $680(\mathrm{P}=\mathrm{S})$.

$\mathrm{H}^{1} \mathrm{NMR}:\left(\mathrm{CDCl}_{3}, 500 \mathrm{MHz}\right)$ ( $\left.\delta: \mathrm{ppm}\right): 1.79-1.76$ $\left(\mathrm{m}, 2 \mathrm{H}, J=2.3, \mathrm{CH}_{2}\right), 1.90-1.88(\mathrm{~m}, 2 \mathrm{H}, J=2.3$ , $\left.\mathrm{CH}_{2}\right), 2.51-2.86\left(2 \mathrm{t}, 4 \mathrm{H}, J=3.5,2 \mathrm{CH}_{2}\right), 3.41,3.45$ $\left[2 \mathrm{~s}, 6 \mathrm{H},\left(\mathrm{OCH}_{3}\right)_{2}\right], 4.52\left(\mathrm{~d}, 1 \mathrm{H},{ }^{3} J_{H P}=4.4, \mathrm{HC}-\mathrm{S}-\mathrm{P}\right)$, $6.70-6.67(\mathrm{~d}, 2 \mathrm{H}, J=8.4$, aromatic $), 7.53(1 \mathrm{H}, \mathrm{s}$, aromatic), 7.74-7.75(d, $2 \mathrm{H}, J=8.5$, aromatic), 7.767.77 (m, 3H, aromatic).

${ }^{13} \mathrm{C}$ NMR $\left(\mathrm{CDCl}_{3}, 125 \mathrm{MHz}\right)(\delta: \mathrm{ppm}): 28.1$ $\left(\mathrm{d},{ }^{2} J_{C P}=29.02\right.$, HC-S-P), 22.9, 24.8, $23.9\left(\mathrm{CH}_{2}\right)$, $53.7\left(\mathrm{OCH}_{3}\right), 112.7,125.8,127.1,127.8,128.9$, 131.0, 132.6, 160.0 (C-aromatic).

Anal. Calc for $\mathrm{C}_{21} \mathrm{H}_{23} \mathrm{O}_{2} \mathrm{PS}_{4} \mathrm{C}, 54.05 \%, \mathrm{H}, 4.97 \%$, $\mathrm{P}, 6.64 \%, \mathrm{~S}, 27.49 \%$ Found : C, $54.22 \%, \mathrm{H}, 4.79 \%$, $\mathrm{P}, 6.61 \%, \mathrm{~S}, 27.40 \%$.

\section{1,9-[4-(Dimethyamino)phenyl]-7,14- dithiadispiro[5.1.5.1]tetradecane $4 a$}

Eluent solution : ethyl acetate / petroleum ether (40$\left.60{ }^{\circ} \mathrm{C}\right) ; 40: 60(\mathrm{v} / \mathrm{v})$.

Yield: $30 \% ; \mathrm{mp}=153^{\circ} \mathrm{C}$.

IR (KBr) v, cm ${ }^{-1}: 2994,2845\left(\mathrm{CH}_{2}\right.$ stretching), 1630 (C=C- stretching), $1225\left(\mathrm{CH}_{2} \mathrm{SCH}_{2}\right.$ stretching), 1609 (=CH stretching).

$\mathrm{H}^{1} \mathrm{NMR}\left(\mathrm{CDCl}_{3}, 500 \mathrm{MHz}\right)$ ( $\left.\delta: \mathrm{ppm}\right): 1.89-1.88$ $\left(\mathrm{m}, 4 \mathrm{H}, 2 \mathrm{CH}_{2}\right), 1.65-1.57\left(\mathrm{~m}, 4 \mathrm{H}, 2 \mathrm{CH}_{2}\right), 2.92(\mathrm{t}$, $\left.4 \mathrm{H}, J=3.0,2 \mathrm{CH}_{2}\right), 2.52\left(\mathrm{t}, 4 \mathrm{H}, J=3.0,2 \mathrm{CH}_{2}\right)$, $3.01,3.10\left[s, 12 \mathrm{H}, 2 \mathrm{~N}\left(\mathrm{CH}_{3}\right)_{2}\right], 6.71,6.68(2 \mathrm{~d}, 4 \mathrm{H}$, $J=8.4$, aromatic), 7.45,7.43 (2d, $4 \mathrm{H}, J=8.4$, aromatic), 7.75(s, $1 \mathrm{H},=\mathrm{CH}), 7.78(\mathrm{~s}, 1 \mathrm{H},=\mathrm{CH})$.

${ }^{13} \mathrm{C}$ NMR: $\left(\mathrm{CDCl}_{3}, 125 \mathrm{MHz}\right)$ ( $\left.\delta: \mathrm{ppm}\right): 22.9$, 24.4, 42.4 $\left(\mathrm{CH}_{2}\right), 35.0$ (spiro-C), 39.3 $\left(\mathrm{N}-\mathrm{CH}_{3}\right), 112.7$, $125.8, \quad 127.1,127.8,128.9,131.0,132.6,148.0$ (C- aromatic).

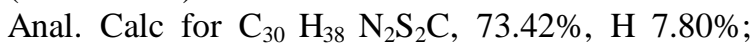
N, $5.71 \%, \mathrm{~S}, 13.07 \%$. Found: C ,73.79\%, H,7.68\%, $\mathrm{N}, 5.91 \%, \mathrm{~S}, 13.32 \%$.

\section{1,9-[4-(Chloro)phenyl]-7,14-} dithiadispiro[5.1.5.1] tetradecane $4 \mathrm{~b}$

Eluent solution : ethyl acetate / petroleum ether (40-60 $\left.{ }^{0} \mathrm{C}\right) ; 35: 65$ (v/v).

Yield; $30 \% ; \mathrm{mp}=171^{\circ} \mathrm{C}$. 
FT-IR (KBr) v, cm-1: 2920, 2850 $\left(\mathrm{CH}_{2}\right.$, stretching), 1530, 1460 (C=C-, stretching), $1230\left(\mathrm{CH}_{2} \mathrm{SCH}_{2}\right.$, stretching), $1620(=\mathrm{CH})$.

$\mathrm{H}^{1} \mathrm{NMR}\left(\mathrm{CDCl}_{3}, 500 \mathrm{MHz}\right)$ ( $\left.\delta: \mathrm{ppm}\right): 1.89-$ $1.88\left(\mathrm{~m}, 2 \mathrm{H}, \mathrm{CH}_{2}\right), 1.65-1.57\left(\mathrm{~m}, 2 \mathrm{H}, \mathrm{CH}_{2}\right), 2.92$ $\left(\mathrm{t}, 4 \mathrm{H}, J=3.0,2 \mathrm{CH}_{2}\right), 3.01\left[\mathrm{~s}, 6 \mathrm{H}, \mathrm{N}\left(\mathrm{CH}_{3}\right)_{2}\right], 6.71$, $6.68(2 \mathrm{~d}, 4 \mathrm{H}, J=8.4$, aromatic), 7.45-7.43 $(2 \mathrm{~d}, 4 \mathrm{H}$, $J=8.4$, aromatic $), 7.75(\mathrm{~s}, 1 \mathrm{H},=\mathrm{CH}), \quad 7.80$ (s, 1H, =CH);

${ }^{13} \mathrm{C} \mathrm{NMR}\left(\mathrm{CDCl}_{3}, 125 \mathrm{MHz}\right) \quad(\delta: \mathrm{ppm}): 22.9$, 24.4, 24.8, 42.4 $\left(\mathrm{CH}_{2}\right), 35.0$ (spiro-C) ,112.7, 125.8,

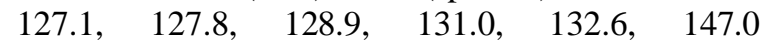
(C-aromatic).

Anal. Calc for $\mathrm{C}_{26} \mathrm{H}_{26} \mathrm{Cl}_{2} \mathrm{~S}_{2} \mathrm{C}, 65.95 \%, \mathrm{H}, 5.53 \%$ ,Cl, $14.97 \%$, S, $13.54 \%$. Found: C, $65.79 \%, \mathrm{H}, 5.62 \%$, $\mathrm{Cl}, 14.81 \%, \mathrm{~S}, 13.32 \%$.

\section{1,9-[2,5-(Dimethoxy)phenyl]-7,14-} dithiadispiro[5.1.5.1]tetradecane 4c

Eluent solution : ethyl acetate / petroleum ether (40-60 $\left.{ }^{0} \mathrm{C}\right) ; 30: 70(\mathrm{v} / \mathrm{v})$.

Yield: $30 \%$; $\mathrm{mp}=160{ }^{\circ} \mathrm{C}$.

FT-IR (KBr) v, cm-1: 2960, $2845\left(\mathrm{CH}_{2}\right.$ stretching );1220 ( $\mathrm{CH}_{2} \mathrm{SCH}_{2}$ stretching); 1609 ( =CH) ;1660 $(\mathrm{C}=\mathrm{C}-$, stretching of aromatic ring).

$\mathrm{H}^{1} \mathrm{NMR}\left(\mathrm{CDCl}_{3}, 500 \mathrm{MHz}\right)$ ( $\left.\delta: \mathrm{ppm}\right): 1.89-1.88$ $\left(\mathrm{m}, 4 \mathrm{H}, 2 \mathrm{CH}_{2}\right), 1.65-1.57\left(\mathrm{~m}, 4 \mathrm{H}, 2 \mathrm{CH}_{2}\right), 2.92$ (t, $\left.4 \mathrm{H}, J=3.4,2 \mathrm{CH}_{2}\right), 2.52\left(\mathrm{t}, 4 \mathrm{H}, J=3.4,2 \mathrm{CH}_{2}\right)$, $3.45, \quad 3.40\left[2 \mathrm{~s}, 12 \mathrm{H}, 2\left(\mathrm{O}-\mathrm{CH}_{3}\right)_{2}\right], \quad 3.56, \quad 3.53$ $\left[2 \mathrm{~s}, 12 \mathrm{H}, 2\left(\mathrm{OCH}_{3}\right)_{2}\right], 6.71,6.68(\mathrm{~d}, 2 \mathrm{H}, J=8.4$, aromatic),7.35, $7.40(2 \mathrm{~s}, 2 \mathrm{H}$, aromatic), 7.45,7.43 (d, 2H, $J=8.4$, aromatic),7.75 (s, $1 \mathrm{H},=\mathrm{CH}), 7.79$ (s, $1 \mathrm{H},=\mathrm{CH})$.

${ }^{13} \mathrm{C}$ NMR $\left(\mathrm{CDCl}_{3}, 125 \mathrm{MHz}\right)$ ( $\left.\delta: \mathrm{ppm}\right): 22.6,24.8$, 42.4 $\left(\mathrm{CH}_{2}\right), \quad 35.7$ (spiro-C), 56.3 $\left(\mathrm{O}-\mathrm{CH}_{3}\right), \quad 112.7$, $\begin{array}{llll}125.8, & 127.1, \quad 127.8,128.9, & 131.0, & 132.6,162.0\end{array}$ (C-aromatic).

Anal. Calc for $\mathrm{C}_{30} \mathrm{H}_{36} \mathrm{O}_{4} \mathrm{~S}_{2} \mathrm{C}, 68.67 \%, \mathrm{H}, 6.92 \%$, S, $12.22 \%$. Found: C, $68.59 \%, \mathrm{H}, 6.86 \%, \mathrm{~S}$, $12.15 \%$.

\section{4-(5,6,7,8-Tetrahydro-2-(4-methoxyphenyl)-2- sulfanyl-4H-benzo[d][1,3,2] dithiaph- sphinin-4-yl)-N,N-dimethylbenzenamine 5a}

Eluent solution : ethyl acetate / petroleum ether $\left(40-60{ }^{0} \mathrm{C}\right) ; 70: 30(\mathrm{v} / \mathrm{v})$.

Yield $62 \% ; \mathrm{mp}=168^{\circ} \mathrm{C}$.

FT-IR (KBr) v, cm-1: 3060, 3015(C-H stretching of aromatic ring) ; 2964, $2840 \quad\left(\mathrm{CH}_{2}\right.$ stretching $)$ ;1660(C $=\mathrm{C}$ stretching) $; 675(\mathrm{P}=\mathrm{S})$.

$\mathrm{H}^{1} \mathrm{NMR}\left(\mathrm{CDCl}_{3}, 500 \mathrm{MHz}\right)$ ( $\left.\delta: \mathrm{ppm}\right): 1.71-1.63$ $\left(\mathrm{m}, 2 \mathrm{H}, \mathrm{CH}_{2}\right), 1.95-1.81\left(\mathrm{~m}, 2 \mathrm{H}, \mathrm{CH}_{2}\right), 2.01,2.48$ $\left(2 \mathrm{t}, 4 \mathrm{H}, J=3.2,2 \mathrm{CH}_{2}\right), 3.00\left[\mathrm{~s}, 6 \mathrm{H}, \mathrm{N}\left(\mathrm{CH}_{3}\right)_{2}\right], 3.5$ (s, 3H, O- $\left.\mathrm{CH}_{3}\right) 4.21\left(\mathrm{~d}, 1 \mathrm{H}, \mathrm{CH},{ }^{3} J_{H P}=3.9\right.$, HC-SP), 6.70,6.67 (2d , 4H, $J=8.4$, aromatic), 7.40,7.38 (2d, $4 \mathrm{H}, J=8.4 \mathrm{~Hz}$, aromatic).

${ }^{13} \mathrm{C}$ NMR: $\left(\mathrm{CDCl}_{3}, 125 \mathrm{MHz}\right) \quad(\delta: \mathrm{ppm}): 34.1$ $\left(d, J_{C P}=24.8 \mathrm{~Hz}, \mathrm{HC}-\mathrm{S}-\mathrm{P}\right), 54.0\left(\mathrm{p}-\mathrm{OCH}_{3}\right), 22.9$, 24.4, 24.8, $27.4\left(\mathrm{CH}_{2}\right), 39.3\left(\mathrm{~N}-\mathrm{CH}_{3}\right), 112.7,125.8$, $127.1, \quad 127.8, \quad 128.9, \quad 131.0, \quad 132.6, \quad 162.0$ (C-aromatic).
Anal. Calc for $\mathrm{C}_{22} \mathrm{H}_{26} \mathrm{NO} \mathrm{PS}_{3} \mathrm{C}, 59.03 \%$, $\mathrm{H} \quad 5.85 \%, \mathrm{~N}, 3.13 \%, \mathrm{P}, 6.92 \%, \mathrm{~S}, 21.49 \%$; Found: C, 59.45\%, H, 5.65\% N, 3.43\%, P, 6.72\%, S, $21.61 \%$.

4-(4-Chlorophenyl)-2-(4-methoxyphenyl)-5,6,7,8tetrahydro-4H-1,3,2-benzodithiaphosphin-ine-2sulfide $\mathbf{5 b}$

Eluent solution : ethyl acetate / petroleum ether (40-60 $\left.{ }^{0} \mathrm{C}\right) ; 75: 25$ (v/v).

Yield: $62 \%: \mathrm{mp}=179^{\circ} \mathrm{C}$;

FT-IR (KBr) v, cm-1: 2964; $2840\left(\mathrm{CH}_{2}\right.$ stretching );760(C-Cl); $660(\mathrm{P}=\mathrm{S})$.

$\mathrm{H}^{1} \mathrm{NMR}\left(\mathrm{CDCl}_{3}, 500 \mathrm{MHz}\right) \quad(\delta: \mathrm{ppm}): 1.61-1.63$ $\left(\mathrm{m}, 2 \mathrm{H}, \mathrm{CH}_{2}\right), 1.85-1.81\left(\mathrm{~m}, 2 \mathrm{H}, \mathrm{CH}_{2}\right), 2.01,2.40$ $\left(2 \mathrm{t}, 4 \mathrm{H}, J=3.8,2 \mathrm{CH}_{2}\right), 3.20\left(\mathrm{~s}, 3 \mathrm{H}, \mathrm{OCH}_{3}\right), 4.21(\mathrm{~d}$, $\left.1 \mathrm{H},{ }^{3} J_{H P}=3.9, \mathrm{CH}-\mathrm{S}-\mathrm{P}\right), 6.70,6.67(2 \mathrm{~d}, 4 \mathrm{H}, J=8.4$, aromatic), 7.40, 7.38 (2d, $4 \mathrm{H} J=8.4$, aromatic);

${ }^{13} \mathrm{C}$ NMR: $\left(\mathrm{CDCl}_{3}, 125 \mathrm{MHz}\right) \quad(\delta: \mathrm{ppm}): 39.3(\mathrm{~d}$, $\left.J_{C P}=34.7 \mathrm{~Hz}, \mathrm{HC}-\mathrm{S}-\mathrm{P}\right), 52.1\left(\mathrm{p}-\mathrm{OCH}_{3}\right), 23.1,24.4$, 24.8, $24.4\left(\mathrm{CH}_{2}\right), 112.7,125.8,127.1,127.8,128.9$, 132.6, 141.7, 161.9 (C-aromatic).

Anal. Calc. for $\mathrm{C}_{20} \mathrm{H}_{20} \mathrm{ClOPS}_{3}$ : C, 54.72\%, H 4.59\%, $\mathrm{Cl}, 8.08 \%$, P, $7.06 \%$, S, $21.91 \%$, Found: C, 54.97\%, $\mathrm{H}, 4.41 \%, \mathrm{Cl}, 8.23 \%, \mathrm{P}, 7.22 \%, \mathrm{~S}, 21.76 \%$.

\section{Biological Methods:}

The antibacterial and antifungal activities were carried out in the Microbial Department, National Research centre, using the diffusion plate method. A solution of each of the tested compound $(5,2.5,1$ $\mathrm{mg} / \mathrm{ml}$ ) was placed onto sterile $5 \mathrm{~mm}$ filter paper discs and allowed to dry, then the discs were placed onto a plate $(9 \mathrm{~cm}$ diameter) containing a solid bacterial medium (nutrient agar) or a fungal medium ( potato dextrose agar) which has been seeded with the spore suspension of the test organism. After incubation at $37^{\circ} \mathrm{C}$ for $24 \mathrm{~h}$ for bacteria (in case of fungi, at $25^{\circ} \mathrm{C}$ for $72 \mathrm{~h}$ ), the diameter of the clear zone of inhibition surrounding the sample is taken as a measure of the inhibitory power of the sample against the particular test organism $(\%$ inhibition $=$ sample inhibition zone (cm) / plate diameter x 100). All measurements were done in methanol as a solvent which has zero inhibition activity. The antimicrobial activities of the tested compounds were examined with Gram-positive bacteria, Bacillus cereus, Staphylococcus aureus ATCC 6538, Salmonella typhimurium ATCC 25566 and Gramnegative bacteria Escherichia coli NRRN 3008 , Pseudomonas aeruginose ATCC 10145 and fungus Candida albicans EMCC105. The obtained results are compared with the Cephradine antibiotics that were purchased from Egyptian markets. ${ }^{28-31}$ 


\section{Results and Discussion}

Reaction of equimolar quantities of 2-[4dimethylaminobenzylidene]cyclohexan-1-one (1a), and 2,4-bis (thiophenoxy)-1,3,2,4- dithiadiphosphetane -2,4-disulfide (JR, 2a) in refluxing toluene afforded a mixture of benzodithiaphosphinin-2 sulfide (3a) and dithiadispiro-tetradecane (4a) (Scheme 2). Compounds 3b,c and 4b,c were similarly produced through reaction of $\mathbf{1 b}, \mathbf{c}$ and $\mathbf{2 a}$. Meanwhile, arylidenecyclohexanones $(\mathbf{1 a}, \mathbf{b})$ reacted with 2,4-bis (methoxyphenyl)-1,3,2,4- dithiadiphosphetane -2,4-disulfide (LR, 2b) in 1:1 molar ratio in boiling toluene giving the respective dithiaphosphetan-2,4-disulfides (5a,b).

The structure of 4[4-(dimethyamino)phenyl]5,6,7,8-tetrahydro-4H-1,3,2-benzodithiaphos-phinin2-sulfide 3a is deduced from its spectroscopic data. ${ }^{1} \mathrm{H}$ NMR spectrum of $\mathbf{3 a}$ exhibits the P-S-CH as a doublet signal $\left(\delta_{\mathrm{H}}=4.39 \mathrm{ppm},{ }^{3} J_{H P}=4.4 \mathrm{~Hz}\right)$. The remaining methylene protons of benzodithiaphosphinin system were revealed at multiplet signals at $\delta_{\mathrm{H}}=1.79,1.90,2.51,2.86 .{ }^{16,20-24}{ }^{13} \mathrm{C} \mathrm{NMR}$ spectrum of 3a shows the P-S-CH- at $\delta_{\mathrm{C}}=34.1 \mathrm{ppm}$. IR spect-rum of $\mathbf{3 a}$ reveals bands at $v=680(\mathrm{P}=\mathrm{S})$, $2910\left(\mathrm{CH}_{2}\right)$ and $1170(\mathrm{P}-\mathrm{S}-) \mathrm{cm}^{-1}{ }^{22}$. Structures of 3b-c and 4a-c have also been supported by spectral data $\left({ }^{1} \mathrm{H}-,{ }^{13} \mathrm{C}\right.$ - NMR, Mass, IR). Structures of dithiaphosphininyldimethylbenzenamine (5a) and dithiaphosphinine-2-sulfide (5b) are confirmed by the presence of molecular ion peaks in their mass spectra $\mathrm{m} / \mathrm{z} 447$ and 438 , respectively, in addition to compatible ${ }^{1} \mathrm{H}-$ and ${ }^{13} \mathrm{C}$ - $\mathrm{NMR}$ spectral data (Experimen-tal) $^{20,21}$.

The reaction proceeded via thiation of compounds 1a,b due to the effect of dithiadiphosphetane-2,4-disulfide $\mathbf{2}$ forming the unstable thioxo intermediates, followed by nucleophilic attack of the sulphide anion of $\mathbf{2 a}, \mathbf{b}$, and cyclization affording isolated $\mathbf{3 a - c}$ and $\mathbf{5 a - b}$ (Scheme 2). Whereas, formation of dithiadispiro [5.1.5.1] tetradecanes 4a-c is due to dimerization of the dipolar form of $\mathbf{1 a}$.<smiles>[R]c1cc(/C=C2\CCCCC2=S)c([R])cc1/C=C1\CCCCC1=O</smiles>

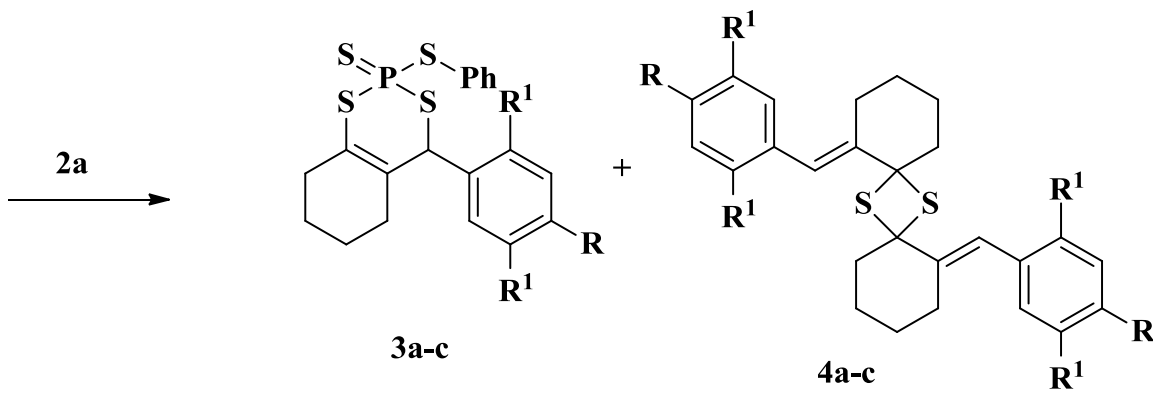

$$
\begin{aligned}
& \text { 3a, } 4 a ; R=N\left(C_{3}\right)_{2}, R^{1}=H \\
& 3 b, 4 b ; R=C l, R^{1}=H \\
& 3 c, 4 c ; R=H, R^{1}=O_{3}
\end{aligned}
$$

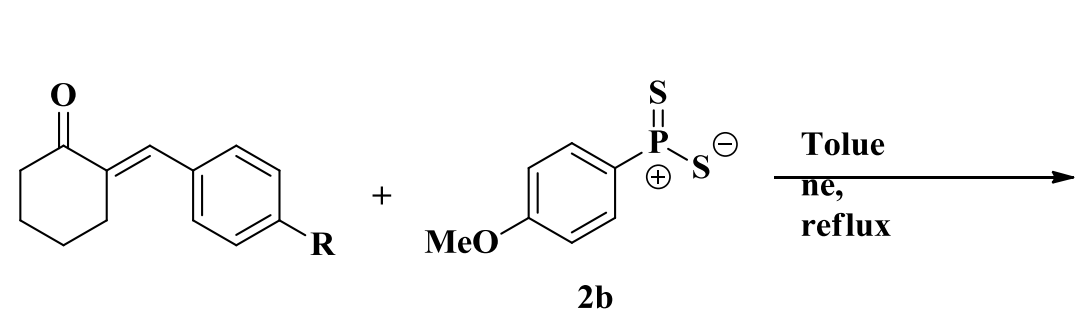

1a; $\mathbf{R}=\mathbf{N}\left(\mathrm{CH}_{3}\right)_{2}$

$\mathbf{1 b} ; \mathbf{R}=\mathbf{C l}$<smiles>[R]c1ccc(C2SP(=S)(c3ccc(OC)cc3)SC3=C2CCCC3)cc1</smiles>

5a; $\mathbf{R}=\mathbf{N}\left(\mathrm{CH}_{3}\right)_{2}$

$\mathbf{5 b} ; \mathbf{R}=\mathbf{C l}$

Scheme 2. Mechanism of 3a-c, 4a-c and 5a, b 


\section{Antimicrobial properties}

The Antimicrobial activities of compounds 3a-c and $\mathbf{5 a}, \mathbf{b}$ were investigated against Gram-positive Gram- negative bacteria and yeast. The results (Table 1) show that the compounds are highly effective against the negative bacteria E-coli,
Pseudomonas aeruginose, and Salmonella sp. with inhibitory power exceeding or equals to that of the reference antibiotic (Cephradine), weak activity against the yeast (Candida albicans). On the other hand moderate antibacterial effect was achieved against Gram-positive bacteria.

Table 1. The antibacterial and antifungal activity of the synthesized compounds

\begin{tabular}{|c|c|c|c|c|c|c|c|}
\hline \multirow[t]{3}{*}{ Compd. } & \multirow[b]{3}{*}{$\mathrm{C}^{*}$} & \multicolumn{6}{|c|}{ Zone of inhibition in $\mathbf{~ m m}$} \\
\hline & & \multicolumn{3}{|c|}{ Gram negative bacteria } & \multicolumn{2}{|c|}{ Gram positive bacteria } & \multirow[b]{2}{*}{ Candida } \\
\hline & & $\begin{array}{r}\text { Escherichia } \\
\text { coli }\end{array}$ & $\begin{array}{r}\text { Pseudomonas } \\
\text { aeruginosa }\end{array}$ & $\begin{array}{r}\text { Salmonella } \\
s p\end{array}$ & $\begin{array}{r}\text { Bacillus } \\
\text { cereus }\end{array}$ & $\begin{array}{l}\text { Staphylococ } \\
\text { cus aurous }\end{array}$ & \\
\hline \multirow[t]{3}{*}{ Cephradine } & 5 & 15 & 20 & 15 & 30 & 30 & 40 \\
\hline & 2.5 & 10 & 15 & 8 & 12 & 12 & 20 \\
\hline & 1 & --- & --- & -- & -- & -- & -- \\
\hline \multirow[t]{3}{*}{$3 \mathbf{a}$} & 5 & 11 & 22 & 15 & -- & 25 & 7 \\
\hline & 2.5 & 5 & 10 & -- & -- & 15 & -- \\
\hline & 1 & -- & -- & -- & -- & -- & -- \\
\hline \multirow[t]{3}{*}{$3 b$} & 5 & 12 & 15 & 11 & 10 & 8 & 13 \\
\hline & 2.5 & 6 & 7 & -- & 6 & -- & -- \\
\hline & 1 & -- & -- & -- & -- & -- & -- \\
\hline \multirow[t]{3}{*}{$3 c$} & 5 & 15 & 18 & 20 & 15 & 10 & 14 \\
\hline & 2.5 & 10 & 8 & -- & 7 & --- & -- \\
\hline & 1 & -- & -- & -- & -- & -- & -- \\
\hline \multirow[t]{3}{*}{$5 a$} & 5 & 8 & 13 & 10 & 8 & 7 & -- \\
\hline & 2.5 & -- & -- & -- & -- & -- & -- \\
\hline & 1 & -- & -- & -- & -- & -- & -- \\
\hline \multirow[t]{3}{*}{$5 b$} & 5 & 10 & 14 & 8 & 12 & 10 & 10 \\
\hline & 2.5 & 6 & -- & -- & -- & -- & -- \\
\hline & 1 & -- & -- & -- & -- & -- & -- \\
\hline
\end{tabular}

N.B. Cephradine was used as standard , * Concentration of the sample in $\mathrm{mg} / \mathrm{ml}$; data are from single experiments and the antimicrobial properties of the test compounds are tentative.

\section{Conclusions}

From all the above it can be concluded that, the reaction of 2-(4-arylidene)cyclohexane-1-one derivatives with 1,3,2,4-dithiadiphosphetane- 2,4disulphides, affords new heterocyclic ring systems containing phosphorus moiety. Furthermore dithiadispiro[5.1.5.1] tetradecanes are formed due to dimerization of 2-arylidenecyclohexanthione. The antimicrobial activity of 1,3,2benzodithiaphosphinine-2-sulfide derivatives were examined with Gram-positive bacteria, Bacillus cereus, Staphylococcus aureus and Gram-negative bacteria Escherichia coli ,Pseudomonas aeruginose, Salmonella $s p$ and fungus Candida albicans. Thus 4[4-(dimethylamino)phenyl]-5,6,7,8-tetrahydro-4H1,3,2-benzodithiaphosphinin-2-sulfide, higher concentration ( $5 \mathrm{mg} / \mathrm{ml})$, exhibited maximum activity against Pseudomonas aeruginos., in the mean time 4-[2,5-dimethoxyphenyl]-5,6,7,8tetrahydro-4H-1,3,2-benzodithiaphosphinin-2-sulfide exhibited maximum activity against Salmonella $s p$. Whereas,4-[2,5-dimethoxy-phenyl]-1,3,2-benzodithia-phosphinin-2-sulfide resulted in $50 \%$ inhibition effect of the Cephradine antibiotic against Bacillus cereus.

\section{References}

1- Z. Hsn, S. Vehora, H. Shinokubo, K. Oshima, J. Org. Chem., 2001, 66, 7854; b) S. kobayashi, K. Kakunoto, M. Sugiura, Org. Lett. 2002 , 4, 1319.

2- F. Fulop, G. Bemath, K. Pihlaja, $A d v$. Heterocycl.Chem., 1998, 69, 349.

3- J. Deli, T. Lorand, D. Szabo, A. Faldesi, Pharmazie 1984, 39, 539.

4- M. N. Arsanious, N. K. El- Din, L. S. Boulos, Phos., Sul. and Sili. 2009, 184, 2813.

5- S. Javanshir, M. M. Mojtahedi, J. Eshami, Curr. Chem. Lett. 2014, 3, 63.

6- K Ranjith, L. Balaganesan,, P. Subbu., Synth. Comm. 2009, 39, 3197; b) N. Singh, J. Pandey, A. Yadav, V. Chaturvedi, S. Bhatnagar, A. N. Gaikwad, S. K. Sinba, A. Kumar, 243 P. K. Shukla, R. P. Tripathi, Eur. J. Med. Chem 2009, $44,1705$.

7- W. M. Weber, L. A. Hunsaker, S. F. Abcouwer, L. M. Deck, D. L. Vander Jagt, Bioorg. Med. Chem, 2005, 13, 3811; b) A. Modzelewska, C. Pettit, G. Achanta, E. Davidson , P. Huang, S. R. Khan, Bioorg Med Chem 2006, 14, 3491. 
8- T. P. Robinson, T. Ehlers, R. B. Hubbard, X. Bai , J. L. Arbiser, D. J. Goldsmith, J. P. 248 Bowena, Bioorg. Med. Chem. Lett. 2003, 13, 115.

9- T. P. Robinson, R. B. Hubbard, T. Ehlers, J. L. Arbiser, D. J. Goldsmith, J. P. Bowena, Bioorg. Med. Chem. 2005,13,4007.

10- J. R. Dimmock, M. P. Padmanilagan, G. A. Zello, K. H. Nienaber, T. M. Allen, L. Santos, E. De Clerck, J. Balzarini, E. K. Manavathu, J. P. Stables, Eur. J. Med. Chem 2003, 38169.

11- A. Modzelewska, C. Pettit, G. Achanta, N. E. Davison, P. Haung, S. R. Khan, Bioorg. Med., Chem. 2006, 14, 3491.

12- C. Piantadosi, I. H. Hall, J. L. Irvin, G. L. Carlson, J. Med. Chem. 1973,16, 770.

13- M. Ogawa, Y. Ishii, T. Nakano, S.Irifune, Jpn Kohai Tokkya JP. 632192446 A2 1988 (C.A. 1988, 63, 238034).

14- R. N. Dighore, P. L. Anandgaonker, S. T. Gaikwad, A. S. Rajbhoj, Mater. Sci. Pol., 33 2015, 259, 163.

15- M. N. Arsanious, S. S. Maigali, L. S. Boulos, Phos., Sul. and Sili. 2010, 185, 57.

16- a) C. M. Simpkins, D. A. Hunt, Tetra. Lett. 2013, 54, 3371;b) P. Perjesi, D. Szabo, G. Batta, A. Foldesi Tetra. Lett. 1987, 28 , 571.

17- R. Shabana, S. S. Maigali, S. A. Essawy, S. A. El- Hussieny, M. F. Soliman, Egy. J. Chem. Special Issue (M.Sidky) 2007, 59.

18- N. R. Mohamed, G. A. Elmegeed, H. A. Abd-El Malek, M. Younis, Stero. 2005, 70, 131.
19- P. Venkateswarlu, S. Ch.Venkata, Tetra. Lett., 2004, $45,3207$.

20- D.H. William, I. Fleming In Spectroscopic Methods in Organic Chemistry, Mc Graw- Hill, Maidenhead, UK, 1987, p.55.

21- M. Hesse, M. Meier, B. Zeeh, Spektroskopische Methoden in der Organischem Chemie Thieme Verlag, Stuttgart, Germany,1997, p 57.

22- L. J. Bellamy, The Infrared Spectra of Complex Molecules, Wiley, New York, 1964, p 311.

23- S. Scheibye, R. Shabana , S.O. Lawesson, Tetera. 1982, 38, 993.

24- A. A El-Barbary, R. Shaban ,S. O. Lawesson, Phosphorus, Sulfur and Silicon 1985, 21, 375.

25- B. Deschamps, J. Seyden-Penne, Tetra. 1977, 33, 413.

26- L. S. Boulos, M. N. Arsanious, E. F. Ewies , Phosphorus, Sulfur and Silicon 2009, 184, 275.

27- L. S. Boulos, M. N.Arsanious , E. F. Ewies, Ramzy F., Z. Natur. 2008, 63b, 1211.

28- R.J. Grayer, B.J. Harborne, Phytochem. 1997, 37, 19.

29- O.N. Irobi, M. Moo-Young, W. A. Anderson, Int.J. Pharmacog 1996, 34, 87.

30- E. Jametz, J. L. Melnick, E. A. Aldelberg Review of Medical Microbiology, 5th ed., Lang Medical Publication: Los Altos, CA 1974, 57, 399.

31- D. N. Muanza, B. W. Kim, K. L. Euler, L. Williams, Int. J. Pharmacog. 1994, 32, 337. 